\title{
Investigating the Putative Impact of Odors Purported to Have Beneficial Effects on Sleep: Neural and Perceptual Processes
}

\author{
Rochelle Ackerley ${ }^{1} \cdot$ Ilona $_{\text {Croy }}{ }^{2} \cdot$ Håkan Olausson $^{3} \cdot$ Gaby Badre $^{4}$ \\ Received: 22 November 2018 / Accepted: 21 July 2019 / Published online: 6 August 2019 \\ (C) The Author(s) 2019
}

\begin{abstract}
Introduction Olfaction has an important role in physiological and affective processes, as well as the potential to have profound effects on activities such as sleep and learning. We investigated two commercially manufactured odors ("Deep Sleep" and "Oriental," from This Works) purported to promote sleep, compared with control odor, where we aimed to explore whether neural and behavioral differences existed after odor inhalation.

Methods In a neuroimaging study, 30 healthy participants were exposed to the odors via an olfactometer during functional magnetic resonance imaging (fMRI). In a further behavioral study using 12 chronic insomniacs, we investigated whether the commercial odors showed effects on sleep during a double-blind, randomized home evaluation.

Results In the neuroimaging, the odors were related to activation of olfactory-relevant areas, such as the orbitofrontal cortex, and we found positive connectivity between the piriform cortex and the hippocampus, amygdala, insula, and middle cingulate cortex. Deep Sleep specifically activated the superior temporal gyrus, whereas Oriental activated the caudate. Further, these commercial odors showed some beneficial impact on sleep.

Conclusions The perceptual and neural impacts of the commercial odors showed that olfactory stimulation can potentially aid sleep and modify affective processes in a number of ways.

Implications The present work opens up opportunities for further investigations into how different odors may lead to specific behavioral and physiological modifications, such as their impact on sleep and well-being, which may provide nonpharmacological alternative approaches.
\end{abstract}

Keywords Smell $\cdot$ Olfaction $\cdot$ Affect $\cdot$ Emotion $\cdot$ Well-being $\cdot$ Sleep

\section{Introduction}

For a long time, fragranced substances (odors, aromas, scents, perfumes) have been used for mental, psychological, physiological, and even spiritual purposes to address disorders and aide well-being, through aromatherapy. Olfaction is the most basic and evocative of our senses (Herz 2004), where there is a strong association between odor and emotion, and olfaction

Gaby Badre

gaby.badre@mednet.gu.se

1 Aix Marseille Univ, CNRS, LNSC (Laboratoire de Neurosciences Sensorielles et Cognitives - UMR 7260), Marseille, France

2 Department of Psychotherapy and Psychosomatic Medicine, Medical Faculty, Technical University of Dresden, Dresden, Germany

3 Center for Social and Affective Neuroscience, Linkoping University, Linkoping, Sweden

4 Gothenburg University, Gothenburg, Sweden can have a profound impact on memories and learning (Wilson 2010; Rihm et al. 2014; Arzi et al. 2014). Further, odors can modify neural processes during sleep (e.g., Perl et al. 2016), which opens up opportunities to affect sleep beneficially through olfaction. Hence, it is of interest to explore non-pharmacological approaches and strategies to address health issues associated with modern-day stressors, which can adversely affect sleep and well-being (e.g., the impact of overusing mobile phones; Munezawa et al. 2011; Thomée et al. 2011).

Specific ingredients commonly used in commercial odorants targeting sleep and well-being (e.g., lavender, patchouli) have been found to induce central relaxant and sedative effects (Haze et al. 2002; Ito and Ito 2011; El Alaoui et al. 2017). However, the process through which an odor produces affective responses is relatively unknown, but it is hypothesized to be via the amygdala, through the absorption of pharmacologically active components of an odor and/or its perceptual associations (Fismer and Pilkington 2012). Odors can nevertheless have profound effects, where studies have reported that 
pleasant odor inhalation may have positive psychophysiological impacts (Liljenquist et al. 2010; Haehner et al. 2017). Conversely, it has been found that people who are intolerant of volatile chemicals (e.g., in multiple chemosensitivities) generally suffer from sleep disturbances and health complaints (Baldwin et al. 2004; Persson et al. 2008). Previous works have demonstrated that odors, especially lavender, can aid sleep and well-being in patients having clinical interventions (e.g., Lytle et al. 2014; Muz and Taşc1 2017; Ayik and Özden 2018). A majority of studies in aromatherapy research have used anecdotal and subjective evidence, where objective investigations are relatively few (Fismer and Pilkington 2012). Only a handful of studies have systematically looked at the effects of pleasant odors on sleep quality in healthy humans (e.g., Hardy et al. 1995; Sano et al. 1998; Tanaka et al. 2002) and have found some beneficial impact, yet we know little about the exact relationship between odors and their neural, behavioral, and perceptual effects.

Understanding the mechanisms of odor neuronal processing and perception is important in comprehending the influence of odors on the brain. It is of interest to further explore these processes in humans by comparing neural and perceptual findings, which we explore presently in our two studies. There is a direct connection from olfactory neurons to limbic and memory areas (Gottfried 2006) and the limbic system (including the amygdala) regulates emotions and behaviors (LeDoux 2012). A behavioral response can be elicited directly by a particular odorant, which may also be linked to the memory of that odorant (Soudry et al. 2011; Billot et al. 2017). This route may be used as a way to impact emotions and behavior profoundly and thus may aid in beneficial outcomes, such as restful sleep.

It is debated whether different odors elicit specific patterns of activation in the brain, as humans differ not only in the memories they relate to different odors but also on the level of the expression of olfactory receptor neurons (Verbeurgt et al. 2014; Croy et al. 2015b). However, there is some evidence that olfactory processing is organized according to the affective perception an odor elicits (for a review, see Zou et al. 2016). For example, Anderson et al. (2003) found that the pleasantness of an odor was processed in spatially separate areas of the orbitofrontal cortex (OFC), which plays a large role in emotional processing. They also showed that an unpleasant odor activated the hippocampus, known for its role in memory. Another area of interest in odor processing is the hypothalamus. Oxytocin is produced in the hypothalamus and it is known to be a "social" hormone promoting sleep, well-being, and relaxation (Carter 2014). Oxytocin is released by tactile stimulation (e.g., massage) (Uvnäs-Moberg et al. 2015) and may also be released by olfactory cues (see Croy et al. (2015b) for potential positive effects and Wyatt (2015) for controversies in the area). Hence, it is of interest to specifically look at any impact of odors on the hypothalamus.
Further, odors can act as excitatory sexual input (Verhaeghe et al. 2013; Bendas et al. 2018), where differences between the sexes may exist.

The present study aimed at evaluating the influence of commercially manufactured odors on cortical brain processes and to assess whether these odors correspondingly produced effects on sleep. Two commercially manufactured complex odors, reported to be pleasant and have positive sensory impacts on sleep and relaxation, were selected and compared with a control odor. One odor ("Deep Sleep") is claimed to aid in falling asleep faster, sleeping better, and waking feeling more refreshed. The other commercial odor ("Oriental") is not currently on the market but is being formulated to have similar benefits to Deep Sleep. We hypothesized that all the odors would produce activity in primary olfactory areas and that the complex commercial odors, aimed at promoting sleep and relaxation, should have additional impact on emotionally relevant brain areas and be rated more positively. From the neuroimaging and behavioral studies, we aimed to identify preliminary markers for assessing the wider impact of odors on the brain and sleep, respectively.

\section{Material and Methods}

Two separate experiments were conducted as follows: (1) a comprehensive spatio-functional study using functional magnetic resonance imaging (fMRI) and (2) a small, exploratory behavioral study in order to investigate whether there is any psycho-physiological impact of the presence of an odor during sleep. Informed consent was obtained from all individual participants included in the study, where they all gave their written consent (details per study below) and were remunerated for their participation. The project was approved by the relevant local ethical committees (in Gothenburg and Dresden) and was conducted in accordance with the Declaration of Helsinki. Different groups of participants were used so not to habituate any of the participants to the odors and because each study was conducted in a place due to the availability of the experimental resources. Further, healthy participants were used in the fMRI study to investigate the typical neuronal responses to the commercial odors, whereas in the behavioral study, we wanted to test a clinical angle, where it was more pertinent to use mild-moderate insomniacs to gain differences in the results and see a larger effect (i.e., we knew that the insomniac group already had sleeping issues, to test the efficacy of the odors). In both studies, two commercially manufactured mixtures of odors containing ingredients claiming to improve sleep (see https://www.thisworks.com/ why-this-works-2/clinical-studies) were tested, namely Deep Sleep (containing lavender, vetivert, chamomile, patchouli, 
and ho wood; full ingredients' list from the manufacturer: water, polysorbate 20, Parfum, Lavandula angustifolia (lavender) oil, Pogostemon cablin (patchouli) oil, Cinnamomum camphora (Ho Wood) oil, Vetiveria zizanoides (vetivert) oil, Ormenis Multicaulis (wild chamomile) flower extract, linalool, diazolidinyl urea, geraniol, limonene, iodopropynyl butylcarbamate) and Oriental (containing patchouli, ylang ylang, and olibanum frankincense; full ingredients' list unavailable, due to the odor not being on the market) (from This Works, London, UK). These were compared with a control odor (synthetic lavender in the behavioral study (containing water, PEG-40 hydrogenated castor oil, Parfum (fragrance), sodium benzoate, potassium sorbate, linalool, disodium EDTA, citric acid, limonene, coumarin, eugenol, geraniol) and phenylethyl alcohol (PEA) in the fMRI study). In the fMRI study, PEA was used as it is commonly used in olfaction neuroimaging (e.g., Hummel et al. 2010; Sezille et al. 2013) and provided a well-validated control for brain activation in olfactory areas, for better comparison with the activity produced from the commercial odors. In the behavioral study, we wanted to test the effect of different odors contrasted to a baseline (no odor), where the control was used in a different way as a comparative common odorant. Synthetic lavender was chosen due to its similarity in character to the commercial odors, yet it was a single, pure odorant rather than a complex, multi-fragranced odor.

\section{fMRI Odor Study}

Thirty participants were tested ( 15 females, 15 males; mean age 24 years $\pm 3 \mathrm{SD}$ ). All of them exhibited normal olfactory function regarding threshold, discrimination, and identification as controlled with Sniffin' Sticks test (Burghardt, Germany) (Hummel et al. 1997) using a cutoff of 32 points (Hummel et al. 2007). The fMRI measurement of the blood oxygenation level-dependent (BOLD) signal was performed with a 3-T scanner (Sonata; Siemens, Erlangen, Germany). Three different odors were presented individually to both nostrils during scanning. The same two odors as used in the behavioral study, Deep Sleep and Oriental, were tested, and compared with the control odor PEA.

In order to ensure a subjective iso-intense odor perception, a pretest with various dilutions per odor was conducted in a sample of 15 different participants. Each of the odors was presented three times in a randomized order and the participants rated the odorants' intensity. The averaged ratings per odor were compared with each other with a $t$ test and isointense odor dilutions were chosen for the fMRI presentation. Based on the pretest results, a dilution of $6.25 \%$ (made in 1,2propanediol) was chosen for Deep Sleep and Oriental, and a dilution of $100 \%$ for the control odor in order to achieve isointense perception.

\section{fMRI Procedure}

Each odor was presented in two runs, where the order was pseudo-randomized (e.g., Deep Sleep-Oriental-PEA followed by a block Deep Sleep-PEA-Oriental). Hence, a total of six runs of odor presentation in on-off (inspiration/exhalation) blocks were performed. Each run consisted of continuous odor presentation for $10 \mathrm{~s}$, followed by a baseline of at least $17 \mathrm{~s}$ during which clean air was presented. The exact number of inhalations per run of a single odor depended on the participant's own breathing frequency, but this was $\sim 10$ (over at least $27 \mathrm{~s}$ ). The three individual odors, as well as the clean air, were applied by a computer-controlled olfactometer with respiratory feedback that enabled synchronization between in breathing and odor presentation, which ensured constancy in the airflow, without any odor-correlated changes due to odor concentration changes (cf. Sezille et al. 2013). The airflow rate was set to $21 / \mathrm{min}$. During the baseline periods, clean air was presented at the same airflow rate. The tubes containing the odors were positioned directly in the scanner and were connected to the participant's nose via a 2-mm tube of 1-m length, which enabled direct and fast stimulus presentation. Odors were presented intranasally, on the right side, using an anatomically formed nosepiece and the participants were given instructions to remain calm and breathe normally.

After each run, the participants were asked to verbally rate the intensity of the odor on a scale from 0 (not intense at all) to 5 (very intense). A total of 150 volumes per run were acquired by means of a 38 axial-slice matrix (TR $3000 \mathrm{~ms} / \mathrm{TE} 40 \mathrm{~ms}$, FA 90; matrix $=348 \times 348$ ). Following the fMRI sessions, a T1-weighted image was acquired in the sagittal direction by using a T1-MPR sequence (TR $1890 \mathrm{~ms} / \mathrm{TE} 3.24 \mathrm{~ms}$; matrix = $238 \times 348$ ): This scan was run to allow individualized brain normalization in the later statistical analysis.

\section{fMRI Analyses}

Intensity ratings were analyzed using SPSS 22 (IBM, Armonk, NY). The main effect of odor type (3 levels: Deep Sleep, Oriental, PEA) and repetition (2 levels: run 1, run 2), as well as the interaction effect, was tested in a repeatedmeasures ANOVA. Post hoc tests were computed as $t$ tests for dependent measurements and were Bonferroni corrected for multiple comparisons. Gender effects were investigated (added as a factor), due to the power given through the high number of participants and the equal gender balance.

Neural data analyses of the $2 \times 2 \times 2 \mathrm{~mm}^{3}$ functional images were performed with SPM 12 software (Statistical Parametric Mapping; Wellcome Department of Imaging Neuroscience, Institute of Neurology at University College London (UCL, UK)), implemented in Matlab (version R2015b; The MathWorks, Natick, MA), following spatial pre-processing with the same software (spatial filtering: 
high-pass filter $=128 \mathrm{~Hz}$, registering, realignment, coregistration between functional and structural images, normalization using segmentation procedure, smoothing by means of $6 \times 6 \times 6 \mathrm{~mm}^{3}$ FWHM Gaussian kernel). Motion regressors from the realignment procedure were included in the generation of $t$ maps for each individual, in order to account for potential head movement. A whole-brain analysis was conducted using the default settings applied in SPM $(p<0.001$, uncorrected). Multiple comparison correction was only applied in this conventional way, and although this may be less stringent than other approaches, it is an accepted level in olfactory fMRI analyses, which are complicated due to a reduced signal-to-noise level (Moessnang and Freiherr 2013).

SPM matrices reflecting the differences between odor and baseline (on-off) were calculated for each fragrance block, based on the general linear modeling approach. As the odors were not perceived as iso-intense (as per the pretest) in the scanner, all activations were corrected for the individual intensity rating at the group level by adding the individual intensity ratings as regressors of no interest in the analysis. Analyses were based on $t$ tests with a global height threshold $p<0.001$ for the overall olfactory activation. All activation coordinates are presented in MNI (Montreal Neurological Institute) space. As we were looking for a possible oxytocin release, it was specifically checked whether the odors would be related to enhanced hypothalamic activation. For this purpose, a region-of-interest (ROI) sphere of $6 \mathrm{~mm}^{3}$ was created around the hypothalamic peak activation observed in a previous odor study (3 0 -8; MNI space, Wallrabenstein et al. 2015) and a threshold of $p<0.01$ (uncorrected) was applied. The more liberal threshold was used, as the ROI analysis minimizes the possibility of false-positive responses; however, interpretation should nevertheless be considered with respect to this level.

As outlined in the "Introduction," consistent and distinguishable neural activity for an individual odor is unlikely; however, the odors produced different results in the behavioral study. In order to capture potential differential neural effects of the odors, we performed a connectivity analysis to focus on the temporal correlation of neural responses. The preprocessed data were analyzed with the connectivity toolbox in SPM (nitrc.org/projects/conn). The primary olfactory cortex (piriform cortex) was used as a seed and functional connectivity was computed over each block, as a linear regression between the seed and the rest of the brain with the CONN toolbox, which performs voxel-seed correlations by estimating temporal correlation maps (Whitfield-Gabrieli and Nieto-Castanon 2012). The piriform cortex was defined according to the criteria described by Zelano et al. (2005), using MRIcron (Maldjian et al. 2003). The analysis focused on the right piriform cortex, reflecting the isolateral olfactory processing from right-sided odor presentation. An FDRcorrected statistical threshold of 0.05 was used.

\section{Behavioral Odor Study}

The behavioral study was designed as a first step into probing perceptual effects of these same odors on sleep. A power analysis (using a power and effect size calculator from Ramakrishnan and Holleran on biomath.info) and comparison with similar previous work (Lewith et al. 2005) revealed that for $>80 \%$ power, at least 9 participants should be included for perceptual responses and 12 for actigraphy. We recruited a total of 12 participants ( 8 females, 4 males; mean age 43 years $\pm 12 \mathrm{SD}$ ) with chronic mild-moderate insomnia (Insomnia Severity Index; Morin et al. 2011; $17 \pm 2$ SD) entered a double-blind 3-week period home evaluation study assessing the efficacy of the two commercial odors, compared with a control. Insomniacs were used as they represented a group requiring aid in sleeping, which the commercial odors were meant to target. Chronic insomnia was defined as difficulties in falling asleep and/or maintaining sleep for several nights a week ( $>3$ days) for more than 3 months and having feelings of not being refreshed when waking up in the morning. The level of insomnia was assessed in a Europeanrecognized sleep laboratory (SDS Kliniken, Gothenburg, Sweden), by a certified expert in sleep medicine. If the participants were on medication upon entering the study, this was continued throughout the trial period. If on any medication, the participants should not have changed it 4 weeks prior to commencing the study; otherwise, they had to be healthy. The inclusion criteria were as follows: a normal (self-reported) sense of smell, insomnia for $>3$ months, difficulties falling asleep and/or maintaining sleep, and having no fragrance sensitivity. The exclusion criteria were the presence of illnesses or newly discovered problems ( $<6$ months), for example, sleep apnea, untreated metabolic disorders, psychiatric disorders, or high blood pressure. Participants were selected by advertising at the sleep clinics and through leaflets on boards.

\section{Behavioral Study Procedure}

The three odors (Deep Sleep, Oriental, control) were randomly assigned in a counter-balanced order. Participants slept the first three nights as usual, and for the proceeding four nights, they sprayed the first randomized odor on their pillowcase before bedtime, evaluating their experiences at the end of the period. Following a few "washing" nights (new pillowcase) without an odor, they slept four nights with a second odor, and the same procedure continued with the third odor. Adherence to this and regularity in the participants' sleep-wake scheme was controlled by actigraphy, which detected their movements (e.g., time-to-bed).

The experimental measures included self-report questionnaires and movement (actigraphy) data. For the subjective reports, participants filled out the daily sleep diary, which also included reports on their daytime behavior and sleep 
perception, with free comments about their night's sleep. They were also able to note if there were any environmental changes of importance (e.g., severe meteorological occurrences, moving house). On awakening, they noted their "sleep quality" on a visual analog scale (VAS; with the end-anchors "very good" and "very bad"), as well as on the Karolinska Sleepiness Scale (KSS, Akerstedt and Gillberg (1990); 1, very alert; 9, very sleepy). The participants also filled out a questionnaire (Table 1) about their perceptions. Physiological movement data were collected using wrist actigraphy (Actiwatch; Cambridge Neurotechnology Ltd., Cambridge, UK), consisting of an accelerometer watch that was worn continuously during the study. However, data were only recorded from eight participants ( 6 females and 2 males), as the data from the other participants were not reliable (e.g., gaps in the recordings). Hence, we do not present the actigraphy results, as this part was underpowered.

\section{Behavioral Study Analyses}

The odors were coded so that the experiment and analyses were conducted double blind. The data were analyzed in Prism (version 7; GraphPad, San Diego, CA), where the data were tested for normality. Significant differences were sought at $p<0.05$. Parametric statistics were used to analyze the KSS, and sleep quality measures. For each, an ANOVA was carried out, where post hoc $t$ tests were corrected for multiple comparisons using the false-discovery rate (FDR) two-stage linear step-up procedure of Benjamini et al. (2006). Each odor response was compared with the response at baseline (no odor) and to the three odors. We primarily wanted to investigate whether an odor could aid sleep on various measures, but it was also of interest to compare the effects between the different odors. Non-parametric statistics were used to analyze the questionnaire data, due to violations of normality. Bonferroni-corrected Wilcoxon tests were used to compare whether each odor deviated from the middle of a questionnaire measure (i.e., the value "5," which represented no difference in the perceptual measure). Gender differences were not investigated due to the unequal number of participants.

\section{Results}

\section{fMRI Odor Study}

There was a significant effect of odor type on intensity ratings $(F[2,29]=29.3, p<0.001)$, but no effect of repeated presentation $(F[1,30]=1.6, p=0.21)$, and no significant interaction effect $(F[2,29]=1.6, p=0.20$, Fig. 1$)$. As post hoc tests revealed that both the odors Deep Sleep and Oriental were rated as significantly more intense than the control odor of PEA, intensity ratings were used as a covariate for all further analyses.

The presentation of the odors was related to a clear activation of olfactory-relevant areas, such as the OFC, amygdala, and insula (Fig. 2 and Table 2). Neural response patterns were further found in the middle cingulate. There were no significant differences in neural activation between both presentations of the same odor. Gender effects are stated where found.

\section{Deep Sleep Odor}

Deep Sleep vs Control After the correction for differences in intensity ratings, the Deep Sleep odor was related to higher BOLD signal changes in the thalamus and middle frontal

Table 1 Subjective questions on sleep in the behavioral odor study, after experiencing each odor

$\begin{array}{llr}\text { 1. How did you find your sleep with the odor? } & 1=\text { convenient } & 10=\text { inconvenient } \\ \text { 2. How was your sleep quality with the odor, compared to your typical sleep quality? } 1 \text { = better } & 10=\text { worse } \\ \text { 3. Was your insomnia worse or better with the odor? } & 1=\text { better } & 10=\text { worse } \\ \text { 4. Did you experience any problems with the odor? } & 1=\text { easier } & 10=\text { many } \\ \text { 5. How did you find maintaining sleep with the odor? } & 1=\text { much } & 10=\text { difficult } \\ \text { 6. Did you find it easier to get to sleep with the odor? } & 1=\text { much } & 10=\text { not at all } \\ \text { 7. Do you feel safer with the odor? } & 1=\text { more rested at all } \\ \text { 8. How do you feel in the morning when you woke up? } & 1=\text { much more } & 10=\text { more tired } \\ \text { 9. Did you experience dreams with the odor, as compared to your typical sleep? } & 1=\text { pleasant } & 10=\text { much less } \\ \text { 10. What was the content of your dreams like? } & 1=\text { drowsy } & 10=\text { unpleasant } \\ \text { 11. After waking up with the odor, how did you experience daytime? } & 1=\text { No difficulty, } 2=\text { Headache, } 3=\text { Darkness, } 4=\text { Dizziness, } \\ \text { 12. After waking up with the odor, did you experience any troubles? } & 5=\text { Nausea, } 6=\text { Fatigue, } 7=\text { Other } \\ \text { 13. Did you feel that the odor effect decreased after...? } & 1=1 \text { day, } 2=2 \text { days, } 3=3 \text { days, } 4=\text { It did not last } \\ & & \text { throughout the test period, } 5=\text { No effect from the } \\ \text { beginning } & 1=1 \text { st odor, } 2=2 \text { nd odor, } 3=3 \text { rd odor, } 4=\text { No odor }\end{array}$




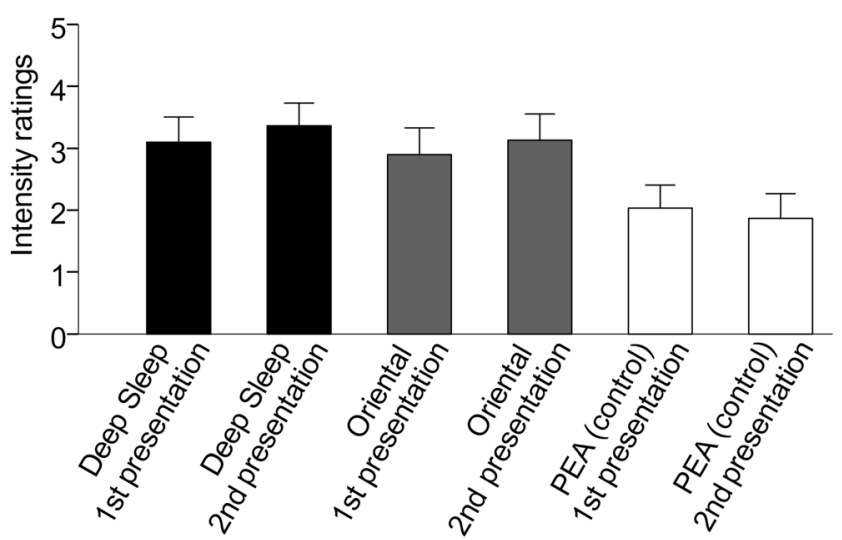

Fig. 1 Intensity ratings within the scanner. Significant effects of odor were observed, but no effect of repetition. The error bars indicate the 95\% confidence intervals

gyrus (Fig. 3 and Table 3). No other brain areas were significantly enhanced for Deep Sleep, compared with the control odor.

Deep Sleep vs Oriental After the correction for differences in intensity ratings, the Deep Sleep odor was related to a higher BOLD signal change in the right superior temporal gyrus.

For the focused hypothalamic activation, Deep Sleep activated this region in women $(t=2.5, p=0.007, k=4)$, but not in men (Fig. 3).

\section{Oriental Odor}

Oriental vs Control After the correction for differences in intensity ratings, the Oriental odor was related to enhanced BOLD signal changes in the right and left caudate, and a cluster which bordered the right thalamus (Fig. 4). None of the primary or secondary olfactory projection areas, and none of the trigeminal projection areas, proved to be significantly enhanced for Oriental compared with the control odor.

Oriental vs Deep Sleep There was no enhanced activation in Oriental odor compared with Deep Sleep. Focusing on hypothalamic activity, Oriental activated this region significantly in men $(t=2.6, p=0.005, k=2)$ but not in women.

\section{Connectivity Analysis}

With each of the odors, the piriform cortex was significantly positively connected to other olfactory and emotionally relevant brain areas, including the hippocampus, anterior insula, and amygdala. The overall connectivity was most widespread and strongest in the control odor (PEA: intensity 170 and 41 nodes; Deep Sleep: intensity 102 and 23 nodes; Oriental: intensity 86 and 18 nodes).

With Deep Sleep, there was a negative connectivity between the piriform cortex and the superior temporal cortex. In Oriental, there was a negative connectivity between the piriform cortex and the posterior cingulate cortex (a node of the default mode network). In the control odor, negative connectivity was detected in the left parietal frontal gyrus (the frontal executive control network) (Fig. 5).

\section{Behavioral Odor Study}

For the perceptual measures, significant differences were found in the KSS measure of alertness (ANOVA $F(3,11)=$ $5.37, p=0.013$ ), where there was significantly increased alertness on waking after sleeping with the Deep Sleep odor compared with baseline sleep (difference $=-1.6 \pm 0.6 \mathrm{SEM}, t=$ $2.86, d f=11, p=0.029)$. There were no significant differences in the same comparisons of baseline sleep compared with the
Fig. 2 Comparison of the neural activation following the "deep sleep," "oriental," and control (PEA) odor. The activations vs. baseline are adjusted for intensity effects and presented in a standardized template with a height threshold of $p<0.001$, uncorrected

\section{Deep Sleep}

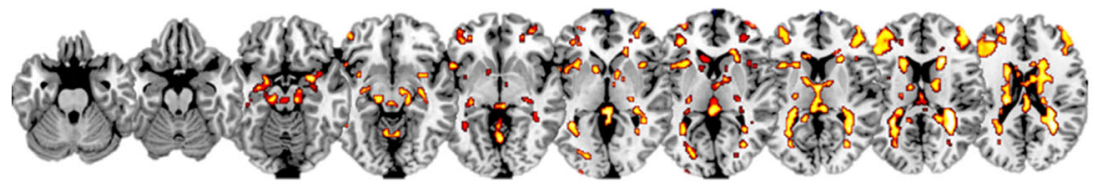

Oriental

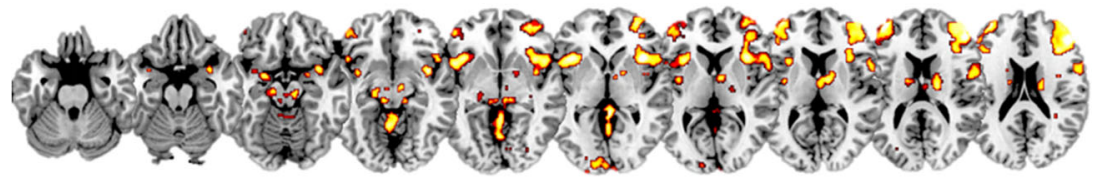

PEA (control)

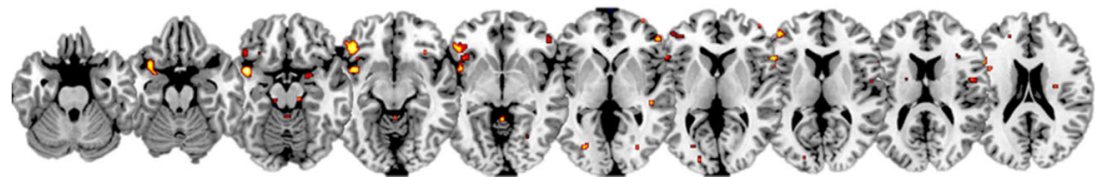


Table 2 Activation to olfactory stimulation (on vs off contrasts), $p<0.001$, uncorrected, cluster extend threshold $k=20$

\begin{tabular}{|c|c|c|c|c|c|}
\hline Cluster size & $T$ & $X$ & $y$ & $z$ & Brain area \\
\hline \multicolumn{6}{|c|}{ "Deep Sleep" odor } \\
\hline 1945 & 5.85 & 39 & 52 & 24 & Right middle frontal gyrus extending to superior temporal gyrus \\
\hline 1420 & 5.26 & -37 & 58 & 14 & Left middle frontal gyrus \\
\hline 960 & 5.38 & 1 & -16 & 12 & Thalamus \\
\hline 564 & 4.04 & 35 & -48 & 36 & Right inferior parietal lobule \\
\hline 553 & 4.68 & -51 & 14 & -4 & Left temporal pole extending to insula \\
\hline 452 & 5.22 & 7 & 18 & 42 & Right middle cingulate cortex \\
\hline 417 & 4.60 & -39 & -48 & 38 & Left inferior parietal lobule \\
\hline 189 & 4.12 & -17 & -94 & 0 & Left middle occipital gyrus \\
\hline 187 & 4.62 & -23 & 0 & -16 & Left amygdala \\
\hline 185 & 4.43 & 17 & -60 & 40 & Right precuneus \\
\hline 157 & 4.46 & 25 & 2 & -16 & Right amygdala \\
\hline 131 & 4.24 & -13 & -8 & 16 & Left caudate nucleus \\
\hline 102 & 3.87 & -5 & -68 & 44 & Left precuneus \\
\hline 102 & 4.52 & 21 & -22 & 22 & Right caudate nucleus \\
\hline 85 & 4.47 & -15 & -20 & -10 & Thalamus \\
\hline 79 & 4.22 & 3 & -98 & 14 & Left cuneus \\
\hline 49 & 4.40 & 59 & -16 & 24 & Right supramarginal gyrus \\
\hline 43 & 3.59 & 29 & -98 & -2 & Right inferior occipital gyrus \\
\hline \multicolumn{6}{|c|}{ "Oriental" odor } \\
\hline 472 & 4.23 & -43 & 20 & -6 & Left inferior frontal gyrus (p. Orbitalis) extending to insula \\
\hline 360 & 4.32 & 19 & 10 & 22 & Right caudate nucleus \\
\hline 166 & 4.50 & 31 & -52 & 22 & Left area hOc1 (V1) \\
\hline 149 & 4.02 & -19 & -58 & 38 & Left middle occipital gyrus extending to superior parietal gyrus \\
\hline 148 & 5.19 & -27 & -74 & 4 & Right area hOc1 (V1) \\
\hline 145 & 4.18 & -23 & 44 & 20 & Left superior frontal gyrus extending to middle frontal gyrus \\
\hline 141 & 3.87 & -49 & 34 & 28 & Left middle frontal gyrus \\
\hline 114 & 3.98 & -35 & 42 & 6 & Left inferior frontal gyrus (p. Triangularis) \\
\hline 42 & 3.77 & 3 & 22 & 38 & Right middle cingulate cortex \\
\hline 40 & 4.15 & 13 & -6 & 12 & Thalamus \\
\hline 38 & 3.86 & 53 & 26 & 30 & Right inferior frontal gyrus \\
\hline 35 & 4.10 & -19 & -22 & 20 & Left caudate nucleus \\
\hline 35 & 3.73 & 45 & 46 & 24 & Right middle frontal gyrus \\
\hline 31 & 3.68 & -45 & 6 & -12 & Left temporal pole extending to insula \\
\hline 26 & 4.10 & -1 & -8 & 8 & Thalamus \\
\hline 25 & 3.79 & 13 & 12 & 0 & Right caudate nucleus \\
\hline 25 & 3.62 & 37 & 28 & -8 & Right inferior frontal gyrus (p. Orbitalis) extending to insula \\
\hline 24 & 3.51 & 43 & 8 & 32 & Right inferior frontal gyrus (p. Opercularis) \\
\hline 21 & 3.79 & -27 & 30 & 6 & Left insula \\
\hline \multicolumn{6}{|c|}{ PEA (control) odor } \\
\hline 293 & 4.36 & 51 & 32 & 30 & Right middle frontal gyrus \\
\hline 191 & 5.17 & 1 & -44 & -4 & Cerebellar vermis $(4 / 5)$ \\
\hline 108 & 4.33 & 45 & -48 & 40 & Right inferior parietal lobule \\
\hline 95 & 4.43 & 47 & 8 & 26 & Right inferior frontal gyrus (p. Opercularis) \\
\hline 93 & 4.32 & 37 & 62 & 2 & Right middle frontal gyrus \\
\hline 43 & 3.88 & -47 & 28 & 38 & Left middle frontal gyrus \\
\hline 32 & 4.28 & 51 & -2 & 14 & Right rolandic operculum \\
\hline 29 & 3.97 & 21 & -72 & 10 & Right calcarine gyrus \\
\hline 21 & 3.80 & 25 & 2 & -16 & Right amygdala \\
\hline 20 & 3.65 & 43 & 30 & 44 & Right middle frontal gyrus \\
\hline 20 & 4.10 & 45 & 10 & -8 & Right insula \\
\hline
\end{tabular}


Fig. 3 Neural activation following the inhalation of the "deep sleep" odor. The images are adjusted for intensity effects and presented in a standardized template. The threshold level is set to $p<0.01$ for visualization purposes
Deep Sleep vs Oriental [whole group]

Deep Sleep vs Control [whole group]
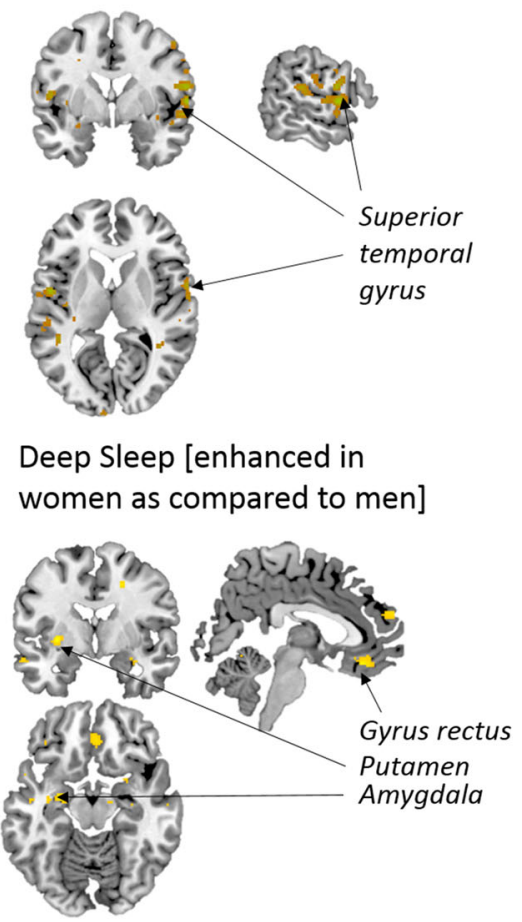

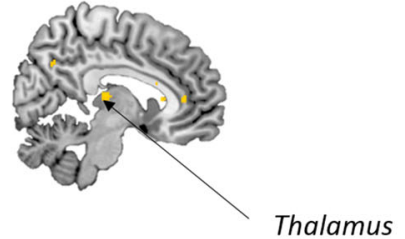

Deep Sleep [only women]

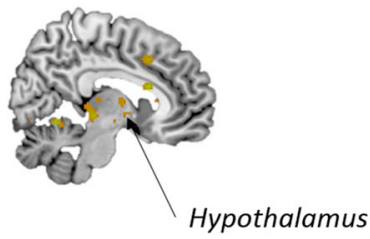

Oriental odor $($ difference $=-0.9 \pm 0.7 \mathrm{SEM}, t=1.30, d f=11$, $p=0.241$ ) or with the control odor (difference $=-0.7 \pm 0.7$ SEM, $t=0.99, d f=11, p=0.359)$. In the sleep quality measure, no significant main effects were found (ANOVA $F(3,11)=0.71, p=0.443)$ and hence, significant differences were not explored between the conditions.

In the subjective questionnaire about the use of the odors (Table 1), we found that Oriental was rated as significantly lower (better) than the middle of the scale (i.e., no difference,

Table 3 Activation in contrasts between odors, $p<0.001$, uncorrected, cluster extend threshold $k=10$ (n.s., no significant activations)

\begin{tabular}{llrrrl}
\hline Cluster size & $T$ & \multicolumn{1}{l}{ x } & \multicolumn{1}{l}{$z$} & Brain area \\
\hline "Deep Sleep" vs control & & & \\
33 & 3.95 & 1 & -16 & 12 & Left thalamus \\
28 & 3.99 & 33 & 36 & 38 & Right middle frontal gyrus \\
"Deep Sleep" vs "Oriental" & & & \\
33 & 3.98 & 65 & -30 & 16 & Right superior temporal gyrus \\
19 & 3.87 & -47 & -30 & 28 & Left supramarginal gyrus \\
16 & 3.60 & 59 & 0 & 16 & Right rolandic operculum \\
13 & 3.85 & 61 & 2 & 6 & Right rolandic operculum \\
11 & 3.96 & -49 & -2 & 10 & Left rolandic operculum \\
"Oriental" vs control & & & & \\
15 & 3.70 & 21 & 18 & 14 & Right caudate nucleus \\
29 & 3.73 & -21 & 16 & 16 & Left caudate nucleus \\
"Oriental” vs Deep Sleep & & & \\
n.s. & & & & & \\
\hline
\end{tabular}

value $=5$ ) on a number of questions, where the participants slept better with this odor present (difference $=-2, W=-28$, $n=12, p=0.044$; question 1), experienced few problems with this odor (difference $=-3, W=-24, n=12, p=0.043$; question 4), and found it easier to get to sleep with this odor (difference $=-1, W=-28, n=12, p=0.016$; question 6), respectively (Fig. 6). There was no clear preference for a particular odor overall, but $75 \%$ of participants preferred sleeping with an odor, rather than no odor. In $58 \%$ of participants, the impact of the odor lasted at least 1 day. None of the odors affected the participants' dreams and occasional headaches and dry mouths were reported.

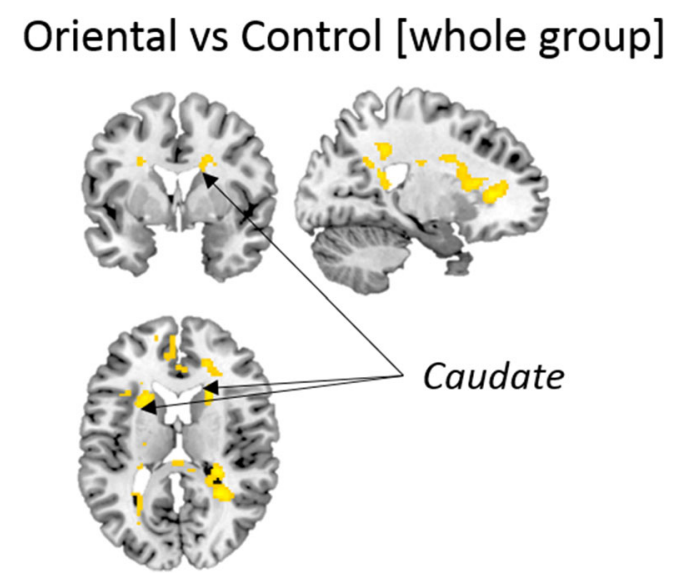

Fig. 4 Neural activation following the inhalation of the "Oriental" odor. The images are adjusted for intensity effects and presented in a standardized template. The threshold level is set to $p<0.01$ for visualization purposes 
Fig. 5 Averaged connectivity results from all of the participants. Positive connectivity is indicated by red lines, and negative connectivity is indicated by blue lines. All results are presented at a threshold of 0.05 , FDR-corrected for multiple comparisons
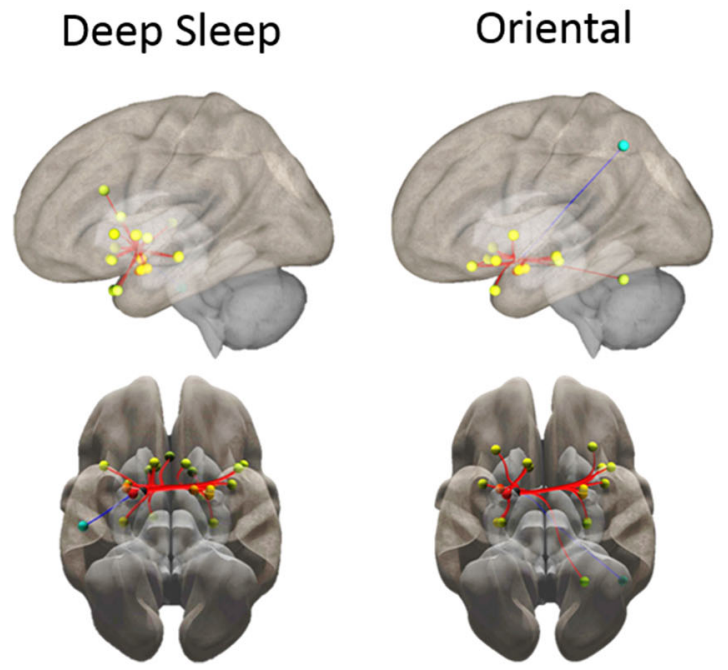

PEA (control)

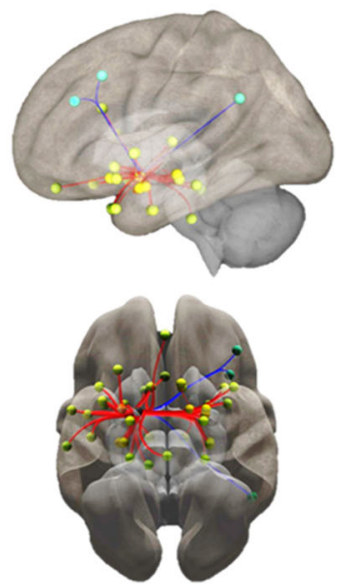

\section{Discussion}

We conducted a comprehensive fMRI study and an exploratory behavioral study to investigate the potential for odors claimed to affect sleep to produce neural and perceptual effects. Our investigation was primarily directed at finding possible ways of measuring how aromatherapy can be used to improve everyday life and well-being. We presented putative data to show that specific neural activity occurred during commercial odor inhalation and that these effects could be assessed using a variety of behavioral approaches. In the fMRI study, all the odors produced significant brain activity in olfactory-related areas, including the cingulate cortex that is related to affective processing of odors (Zou et al. 2016) and the complexity of an odor (Sezille et al. 2015). We found further specific activations for each of the commercial odors tested. For example, with Deep Sleep, the superior temporal gyrus was activated, whereas with Oriental we found additional caudate activity. These additional activations may have occurred for a number of reasons, including the processing of the increased complexity of the commercial odors and the affective consequences of this. However, this interpretation is limited by the possibility that the odors were sniffed with different intensities (although we found a similar inhalation frequency across all the odors) and that the inhalation of the present odors nevertheless produced impacts on neural processing over normal respiration (cf. Koritnik et al. 2009).

The pronounced effect of all the odors on the brain showed that these were well registered within the olfactory system and in further areas, such as the anterior intra-parietal sulcus, which is an area involved in somatosensory integration, likely activated due to stimulation of the trigeminal nerve (cf. Croy et al. 2014). The activation of the middle cingulate cortex that was especially seen with Deep Sleep suggested that the odors evoked emotional responses, which may have influences on behavior (Hadland et al. 2003), although multiple brain areas
Fig. 6 Median scores and interquartile range from questions about sleep (see Table 1).

"Oriental" was rated as significantly lower (better sleep experience) for measures 1,4 , and 6

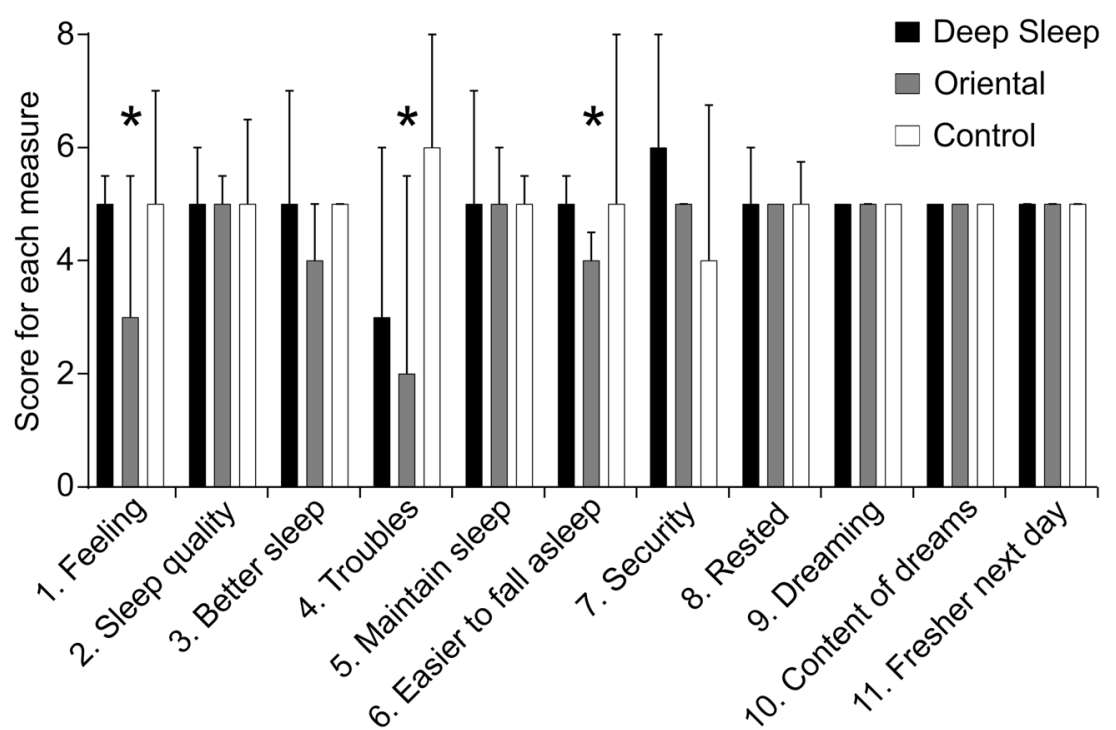


are involved in such affective and motivational processes (LeDoux 2000). The activation of the caudate (Oriental) and the putamen (Deep Sleep) suggested the participation of processes associated with reward or novelty (Báez-Mendoza and Schultz 2013) for the commercial odors, which may relate to the complex nature of the commercial odors and their interpretation. Similarly, Deep Sleep was related to the highest BOLD signal changes in the middle frontal gyrus, an area involved in the top-down modulation of attention (Japee et al. 2015) and Oriental showed higher activation than the control in the caudate, which is an area associated with reward processes (Knutson and Cooper 2005), again likely reflecting the complexity of the commercial odors. As compared with Oriental, Deep Sleep produced stronger activations in the right superior temporal gyrus, an area implicated in social perception and emotion (Narumoto et al. 2001; Zahn et al. 2007). This area was furthermore significantly coupled to the piriform cortex in Deep Sleep, suggesting a direct effect of olfactory areas, stimulated by Deep Sleep on the superior temporal gyrus. Gender-specific effects were observed for the hypothalamus, with enhanced activation in men for Oriental and an enhanced activation in women for Deep Sleep. The result of gender-differentiated hypothalamic activation of odors is in line with previous findings of sex differences in the hypothalamus associated with putative pheromone inhalation (Wallrabenstein et al. 2015), but given the a priori search and activation probability threshold, it would be preferable to conduct further studies into this.

We also a found coupling between the primary olfactory region (piriform cortex) and other olfactory and emotionally relevant brain areas brain regions, including the amygdala that is known for its role in emotion (LeDoux 2012), which was the case across all the odor conditions. Overall, the commercial odors seemed to show more widespread neural activity, yet the connectivity between the primary olfactory region and other brain areas was more focused on the commercial odors than with the control odor. These differences likely represent how the more simple control odor differed in its quality and subsequent processing, as compared with the multi-fragranced commercial odors. The increased complexity of the commercial odors likely required further central processing, where the close relationship between olfactive and affective processing (Weber and Heuberger 2008) may entail the recruitment of more emotional and cognitive structures. The higher an odorant's complexity, the more likely it is to be evaluated as pleasant (Kermen et al. 2011) and plant-derived odors are also typically classed as pleasant and can positively affect states such as calmness, alertness, and mood (Weber and Heuberger 2008). The commercial odors we tested presently were both complex and plant based, and although we did not explicitly test their affective qualities, we found that they were well tolerated during the fMRI and behavioral studies. Moreso, in the behavioral study, we found that the majority of participants preferred sleeping with an odor than without. The pleasantness of an odor may play a role in mediating its effects, including over longer periods, such as during sleep, thus it seems advantageous to both design and test the effects of complex odors.

The presentation of odors for extended time periods leads to their adaptation (Croy et al. 2015a). As there was no effect of repeated odor stimulation, we assumed that the neural effect did not weaken over time in the scanner. Our block design thus appeared to work well, providing a balance between statistical power and the effects of repeated olfactory stimulation. However, the effect of adaptation to odors for longer time periods (e.g., during sleep) should be taken into account, as the neural (and also perceptual) effects may change (Croy et al. 2013), although it is known that odors are processed nevertheless during sleep (e.g., odors can affect dreams; Schredl et al. 2009) and in our behavioral study, 58\% of participants found that the impact of the odor lasted at least 1 day. Therefore, the potential mechanisms underlying the effects of odor inhalation at the beginning of sleep may be through such processes, but we cannot assume these are the same over time, due to the perceptual and/or physiological adaptation of odors, and decreased levels of consciousness during sleep. However, it is likely that the effects of odor inhalation can persist and this may be a way to influence health and well-being for more prolonged periods.

In our additional exploratory behavioral study, we investigated whether the two commercial odors and a single fragrance control odor could impact sleep. We found that the commercial odors were rated as having a positive significant impact on sleep, but that such studies may be limited by the variability between subjects. Specifically, we found that Oriental was rated as producing significantly better overt sleep effects (as measured through the questionnaire about how the odors were perceived) and Deep Sleep produced decreased sleepiness on waking (as measured through the alertness on waking in the KSS). Hence, the more complex commercial odors were found to have beneficial effects in the perceptual ratings; however, it is unknown which aspect(s) of these odors led to this finding (e.g., the content and/or hedonics of the odor). Akin to the fMRI findings, it is likely that the multifragranced commercial odors may have further impacts, where the active ingredients (e.g., lavender, patchouli, vetivert, chamomile) may alter central processes (Haze et al. 2002; Ito and Ito 2011; El Alaoui et al. 2017). Overall, it was clear that the insomniac participants preferred to sleep with an odor, than without, which may mean that simply having such an odor present may aid sleep and restfulness. Comparable beneficial perceptual effects were found in a similar-sized study on insomniacs testing the effects of lavender odor on sleep (Lewith et al. 2005), but we aimed to explore whether actigraphy may prove a useful measure. However, we were not able to include these data as there was variability in our 
actigraphy measures (e.g., missing data due to non-adherence to the protocol) and this part of the study was underpowered, but the current work is a step toward seeing whether such effects are measurable and we believe that a future larger study may yield promising results. An advantage of our approach was that it may be more ecological (e.g., a field study); however, the participant-led method produced increased variability than would be found in more standard approaches (e.g., laboratory testing). Overall, our preliminary findings show the potential for odors to produce both perceptual and behavioral beneficial effects on sleep, which should be investigated further.

\section{Conclusions}

We found that a number of brain areas were activated in our fMRI study, where more areas were recruited during inhalation of the commercial odors and there were differences between the areas activated between the odors. The more profound activations for the commercial odors may relate to their increased olfactory complexity compared with the control (e.g., the number and type of ingredients). We cannot comment on the way in which single chemicals may activate the brain as we investigated blends; however, the differential effects may be due to both lower level basic processing of the specific chemicals and/or the individual perception of these. In our exploratory sleep study, using the same commercial odors, we found it was possible to investigate the impact of odorant inhalation during sleep, where the commercial odors showed beneficial effects on the subjective rating of sleep and its quality. Further studies are needed into such mechanisms, but overall, our present results suggest that different odors may relate to specific perceptual and neuronal alterations. The ingredients in the commercial odors have been chosen due to previous findings that such components (e.g., patchouli, lavender) may induce relaxant and sedative effects, which we presently found some evidence for in the brain activations found and in the perceptual responses.

\section{Implications}

The present work provides findings about psychological and functional activations of odor inhalation. It opens up opportunities for further investigations into how different odors may lead to specific behavioral and physiological modifications on sleep and their impact on well-being, which may provide nonpharmacological alternatives that may be safer than prescribed drugs and more cost-effective.
Funding Information Open access funding provided by University of Gothenburg.

\section{Compliance with Ethical Standards}

Conflict of Interest The studies were initiated by the authors and This Works provided the odors and supported the fMRI study financially. Prof. Badre is an independent expert scientific advisor for This Works, but has no financial role or involvement in the company.

Ethical Approval All procedures performed in studies involving human participants were in accordance with the ethical standards of the institutional and/or national research committee and with the 1964 Helsinki Declaration and its later amendments or comparable ethical standards.

Informed Consent Informed consent was obtained from all individual participants included in the study, where they all gave their written consent and were remunerated for their participation.

Open Access This article is distributed under the terms of the Creative Commons Attribution 4.0 International License (http:// creativecommons.org/licenses/by/4.0/), which permits unrestricted use, distribution, and reproduction in any medium, provided you give appropriate credit to the original author(s) and the source, provide a link to the Creative Commons license, and indicate if changes were made.

\section{References}

Akerstedt T, Gillberg M (1990) Subjective and objective sleepiness in the active individual. Int J Neurosci 52:29-37

Anderson AK, Christoff K, Stappen I, Panitz D, Ghahremani DG, Glover G, Gabrieli JDE, Sobel N (2003) Dissociated neural representations of intensity and valence in human olfaction. Nat Neurosci 6:196202. https://doi.org/10.1038/nn1001

Arzi A, Holtzman Y, Samnon P, Eshel N, Harel E, Sobel N (2014) Olfactory aversive conditioning during sleep reduces cigarettesmoking behavior. J Neurosci 34:15382-15393. https://doi.org/10. 1523/JNEUROSCI.2291-14.2014

Ayik C, Özden D (2018) The effects of preoperative aromatherapy massage on anxiety and sleep quality of colorectal surgery patients: a randomized controlled study. Complement Ther Med 36:93-99. https://doi.org/10.1016/j.ctim.2017.12.002

Báez-Mendoza R, Schultz W (2013) The role of the striatum in social behavior. Front Neurosci 7:233

Baldwin CM, Bell IR, Guerra S, Quan SF (2004) Associations between chemical odor intolerance and sleep disturbances in communityliving adults. Sleep Med 5:53-59. https://doi.org/10.1016/S13899457(03)00164-3

Bendas J, Hummel T, Croy I (2018) Olfactory function relates to sexual experience in adults. Arch Sex Behav 47:1333-1339. https://doi. org/10.1007/s10508-018-1203-X

Benjamini Y, Krieger A, Yekutieli D (2006) Adaptive linear step-up procedures that control the false discovery rate. Biometrika 93:491-507

Billot PE, Andrieu P, Biondi A, Vieillard S, Moulin T, Millot JL (2017) Cerebral bases of emotion regulation toward odours: a first approach. Behav Brain Res 317:37-45. https://doi.org/10.1016/j.bbr. 2016.09.027

Carter CS (2014) Oxytocin pathways and the evolution of human behavior. Annu Rev Psychol 65:17-39. https://doi.org/10.1146/annurevpsych-010213-115110 
Croy I, Krone F, Walker S, Hummel T (2015a) Olfactory processing: detection of rapid changes. Chem Senses 40:351-355. https://doi. org/10.1093/chemse/bjv020

Croy I, Maboshe W, Hummel T (2013) Habituation effects of pleasant and unpleasant odors. Int J Psychophysiol 88:104-108. https://doi. org/10.1016/j.ijpsycho.2013.02.005

Croy I, Olgun S, Mueller L, Schmidt A, Muench M, Hummel C, Gisselmann G, Hatt H, Hummel T (2015b) Peripheral adaptive filtering in human olfaction? Three studies on prevalence and effects of olfactory training in specific anosmia in more than 1600 participants. Cortex 73:180-187. https://doi.org/10.1016/j.cortex.2015.08. 018

Croy I, Schulz M, Blumrich A, Hummel C, Gerber J, Hummel T (2014) Human olfactory lateralization requires trigeminal activation. Neuroimage 98:289-295. https://doi.org/10.1016/j.neuroimage. 2014.05.004

El Alaoui C, Chemin J, Fechtali T, Lory P (2017) Modulation of T-type $\mathrm{Ca} 2+$ channels by lavender and rosemary extracts. PLoS One 12: e0186864. https://doi.org/10.1371/journal.pone.0186864

Fismer KL, Pilkington K (2012) Lavender and sleep: a systematic review of the evidence. Eur J Integr Med 4:e436-e447. https://doi.org/10. 1016/j.eujim.2012.08.001

Gottfried JA (2006) Smell: central nervous processing. In: Advances in Oto-Rhino-Laryngology. KARGER, Basel, pp 44-69

Hadland KA, Rushworth MFS, Gaffan D, Passingham RE (2003) The effect of cingulate lesions on social behaviour and emotion. Neuropsychologia 41:919-931. https://doi.org/10.1016/S00283932(02)00325-1

Haehner A, Maass H, Croy I, Hummel T (2017) Influence of room fragrance on attention, anxiety and mood. Flavour Fragr J 32:24-28. https://doi.org/10.1002/ffj.3339

Hardy M, Kirk-Smith MD, Stretch DD (1995) Replacement of drug treatment for insomnia by ambient odour. Lancet 346:701. https://doi. org/10.1016/S0140-6736(95)92310-1

Haze S, Sakai K, Gozu Y (2002) Effects of fragrance inhalation on sympathetic activity in normal adults. Jpn J Pharmacol 90:247-253. https://doi.org/10.1254/jjp.90.247

Herz RS (2004) A naturalistic analysis of autobiographical memories triggered by olfactory visual and auditory stimuli. Chem Senses 29:217-224. https://doi.org/10.1093/chemse/bjh025

Hummel T, Fliessbach K, Abele M, Okulla T, Reden J, Reichmann H, Wüllner U, Haehner A (2010) Olfactory fMRI in patients with Parkinson's disease. Front Integr Neurosci 4:125. https://doi.org/ 10.3389/fnint.2010.00125

Hummel T, Kobal G, Gudziol H, Mackay-Sim A (2007) Normative data for the "Sniffin' Sticks" including tests of odor identification, odor discrimination, and olfactory thresholds: an upgrade based on a group of more than 3,000 subjects. Eur Arch Oto-RhinoLaryngology 264:237-243. https://doi.org/10.1007/s00405-0060173-0

Hummel T, Sekinger B, Wolf SR, Pauli E, Kobal G (1997) "Sniffin' sticks": olfactory performance assessed by the combined testing of odor identification, odor discrimination and olfactory threshold. Chem Senses 22:39-52

Ito $\mathrm{K}$, Ito M (2011) Sedative effects of vapor inhalation of the essential oil of Microtoena patchoulii and its related compounds. J Nat Med 65: 336-343. https://doi.org/10.1007/s11418-010-0502-x

Japee S, Holiday K, Satyshur MD, Mukai I, Ungerleider LG (2015) A role of right middle frontal gyrus in reorienting of attention: a case study. Front Syst Neurosci 9:23. https://doi.org/10.3389/fnsys.2015. 00023

Kermen F, Chakirian A, Sezille C et al (2011) Molecular complexity determines the number of olfactory notes and the pleasantness of smells. Sci Rep 1:206. https://doi.org/10.1038/srep00206
Knutson B, Cooper JC (2005) Functional magnetic resonance imaging of reward prediction. Curr Opin Neurol 18:411-417. https://doi.org/10. 1097/01.wco.0000173463.24758.f6

Koritnik B, Azam S, Andrew CM, Leigh PN, Williams SCR (2009) Imaging the brain during sniffing: a pilot fMRI study. Pulm Pharmacol Ther 22:97-101. https://doi.org/10.1016/j.pupt.2008. 10.009

LeDoux J (2012) Rethinking the emotional brain. Neuron 73:653-676. https://doi.org/10.1016/j.neuron.2012.02.004

LeDoux JE (2000) Emotion circuits in the brain. Annu Rev Neurosci 23: 155-184. https://doi.org/10.1146/annurev.neuro.23.1.155

Lewith GT, Godfrey AD, Prescott P (2005) A single-blinded, randomized pilot study evaluating the aroma of Lavandula augustifolia as a treatment for mild insomnia. J Altern Complement Med 11:631637. https://doi.org/10.1089/acm.2005.11.631

Liljenquist K, Zhong CB, Galinsky AD (2010) The smell of virtue: clean scents promote reciprocity and charity. Psychol Sci 21:381-383. https://doi.org/10.1177/0956797610361426

Lytle J, Mwatha C, Davis KK (2014) Effect of lavender aromatherapy on vital signs and perceived quality of sleep in the intermediate care unit: a pilot study. Am J Crit Care 23:24-29. https://doi.org/10.4037/ ajcc2014958

Maldjian JA, Laurienti PJ, Kraft RA, Burdette JH (2003) An automated method for neuroanatomic and cytoarchitectonic atlas-based interrogation of fMRI data sets. Neuroimage 19:1233-1239. https://doi. org/10.1016/S1053-8119(03)00169-1

Moessnang C, Freiherr J (2013) Olfaktorik. In: Funktionelle MRT in Psychiatrie und Neurologie. Springer, Berlin Heidelberg, Berlin, Heidelberg, pp 505-521

Morin CM, Belleville G, Bélanger L, Ivers H (2011) The insomnia severity index: psychometric indicators to detect insomnia cases and evaluate treatment response. Sleep 34:601-608. https://doi.org/10. 1093/sleep/34.5.601

Munezawa T, Kaneita Y, Osaki Y, Kanda H, Minowa M, Suzuki K, Higuchi S, Mori J, Yamamoto R, Ohida T (2011) The association between use of mobile phones after lights out and sleep disturbances among Japanese adolescents: a nationwide cross-sectional survey. Sleep 34:1013-1020. https://doi.org/10.5665/SLEEP.1152

Muz G, Tasc1 S (2017) Effect of aromatherapy via inhalation on the sleep quality and fatigue level in people undergoing hemodialysis. Appl Nurs Res 37:28-35. https://doi.org/10.1016/j.apnr.2017.07.004

Narumoto J, Okada T, Sadato N, Fukui K, Yonekura Y (2001) Attention to emotion modulates fMRI activity in human right superior temporal sulcus. Cogn Brain Res 12:225-231. https://doi.org/10.1016/ S0926-6410(01)00053-2

Perl O, Arzi A, Sela L, Secundo L, Holtzman Y, Samnon P, Oksenberg A, Sobel N, Hairston IS (2016) Odors enhance slow-wave activity in non-rapid eye movement sleep. J Neurophysiol 115:2294-2302. https://doi.org/10.1152/jn.01001.2015

Persson R, Eek FC, Österberg K et al (2008) A two-week monitoring of self-reported arousal, worry and attribution among persons with annoyance attributed to electrical equipment and smells. Scand J Psychol 49:345-356. https://doi.org/10.1111/j.1467-9450.2008. 00660.x

Rihm JS, Diekelmann S, Born J, Rasch B (2014) Reactivating memories during sleep by odors: odor specificity and associated changes in sleep oscillations. J Cogn Neurosci 26:1806-1818. https://doi.org/ 10.1162/jocn_a_00579

Sano A, Sei H, Seno H et al (1998) Influence of cedar essence on spontaneous activity and sleep of rats and human daytime nap. Psychiatry Clin Neurosci 52:133-135. https://doi.org/10.1111/j. 1440-1819.1998.tb00991.x

Schredl M, Atanasova D, Hörmann K et al (2009) Information processing during sleep: the effect of olfactory stimuli on dream content and dream emotions. J Sleep Res 18:285-290. https://doi.org/10.1111/j. 1365-2869.2009.00737.x 
Sezille C, Ferdenzi C, Chakirian A, Fournel A, Thevenet M, Gerber J, Hummel T, Bensafi M (2015) Dissociated neural representations induced by complex and simple odorant molecules. Neuroscience 287:23-31. https://doi.org/10.1016/j.neuroscience.2014.12.011

Sezille C, Messaoudi B, Bertrand A, Joussain P, Thévenet M, Bensafi M (2013) A portable experimental apparatus for human olfactory fMRI experiments. J Neurosci Methods 218:29-38. https://doi.org/10. 1016/j.jneumeth.2013.04.021

Soudry Y, Lemogne C, Malinvaud D, Consoli SM, Bonfils P (2011) Olfactory system and emotion: common substrates. Eur Ann Otorhinolaryngol Head Neck Dis 128:18-23. https://doi.org/10. 1016/j.anorl.2010.09.007

Tanaka J, Uchimura N, Hashizume Y et al (2002) Effects of aroma on sleep and biological rhythms. Psychiatry Clin Neurosci 56:299-300. https://doi.org/10.1046/j.1440-1819.2002.00984.x

Thomée S, Härenstam A, Hagberg M (2011) Mobile phone use and stress, sleep disturbances, and symptoms of depression among young adults-a prospective cohort study. BMC Psychiatry 11:66. https://doi.org/10.1186/1471-244X-12-176

Uvnäs-Moberg K, Handlin L, Petersson M (2015) Self-soothing behaviors with particular reference to oxytocin release induced by nonnoxious sensory stimulation. Front Psychol 5:1529. https://oi.org/ 10.3389/fpsyg.2014.01529

Verbeurgt C, Wilkin F, Tarabichi M, Gregoire F, Dumont JE, Chatelain P (2014) Profiling of olfactory receptor gene expression in whole human olfactory mucosa. PLoS One 9:e96333. https://doi.org/10. 1371/journal.pone.0096333

Verhaeghe J, Gheysen R, Enzlin P (2013) Pheromones and their effect on women's mood and sexuality. Facts, views Vis ObGyn 5:189-195

Wallrabenstein I, Gerber J, Rasche S, Croy I, Kurtenbach S, Hummel T, Hatt H (2015) The smelling of Hedione results in sex-differentiated human brain activity. Neuroimage 113:365-373. https://doi.org/10. 1016/j.neuroimage.2015.03.029

Weber ST, Heuberger E (2008) The impact of natural odors on affective states in humans. Chem Senses 33:441-447. https://doi.org/10. 1093/chemse/bjn011

Whitfield-Gabrieli S, Nieto-Castanon A (2012) Conn: a functional connectivity toolbox for correlated and anticorrelated brain networks. Brain Connect 2:125-141. https://doi.org/10.1089/brain.2012.0073

Wilson DA (2010) Single-unit activity in piriform cortex during slowwave state is shaped by recent odor experience. J Neurosci 30:17601765. https://doi.org/10.1523/JNEUROSCI.5636-09.2010

Wyatt TD (2015) The search for human pheromones: the lost decades and the necessity of returning to first principles. Proc R Soc B Biol Sci 282:20142994. https://doi.org/10.1098/rspb.2014.2994

Zahn R, Moll J, Krueger F, Huey ED, Garrido G, Grafman J (2007) Social concepts are represented in the superior anterior temporal cortex. Proc Natl Acad Sci 104:6430-6435. https://doi.org/10.1073/pnas. 0607061104

Zelano C, Bensafi M, Porter J, Mainland J, Johnson B, Bremner E, Telles C, Khan R, Sobel N (2005) Attentional modulation in human primary olfactory cortex. Nat Neurosci 8:114-120. https://doi.org/10. $1038 / \mathrm{nn} 1368$

Zou L q, van Hartevelt TJ, Kringelbach ML et al (2016) The neural mechanism of hedonic processing and judgment of pleasant odors: an activation likelihood estimation meta-analysis. Neuropsychology 30:970-979. https://doi.org/10.1037/neu0000292

Publisher's Note Springer Nature remains neutral with regard to jurisdictional claims in published maps and institutional affiliations. 